\title{
INTEGRACIÓN DE LA ECONOMÍA CON LA PSICOLOGÍA: RICHARD H. THALER, PREMIO NOBEL DE ECONOMÍA 2017
}

\author{
INTEGRATION OF THE ECONOMY WITH PSYCHOLOGY: RICHARD H. \\ THALER, NOBEL PRIZE OF ECONOMY 2017 \\ José G. Vargas-Hernández* \\ enviado: 12 octubre 2017 - aceptado: 22 abril 2018
}

\begin{abstract}
Resumen
Este trabajo pretende analizar las principales contribuciones de Richard H. Thaler a la ciencia de la economía del comportamiento, las cuales han sido consideradas para otorgarle el Premio Nobel Sveriges Riksbank de Economía en 2017. Sus aportaciones incorporan la psicología en los procesos de tomas de decisiones económicas apartándose de la racionalidad instrumental y la teoría de la elección racional para contribuir a la generación de una nueva perspectiva en la teoría económica y en la política económica. El método empleado es fundamentalmente el analítico-descriptivo basado en una revisión de sus principales publicaciones realizadas. Finalmente se concluye que Thaler y sus colaboradores ejercen una influencia relevante al generar un nuevo marco de referencia para explicar y predecir resultados económicos desde un acercamiento del comportamiento racional limitado, la teoría de contabilidad limitada y el modelo de planeador-hacedor bajo el concepto de empujón o nudge, el cual tiene un impacto en el diseño e implementación de las políticas públicas.
\end{abstract}

Código JEL: B31, B41, D91.

Palabras clave: empujón, economía conductual, efecto de dotación, contabilidad mental, racionalidad limitada.

- Vargas-Hernández, J. G. (2018). Integración de la economía con la psicología: Richard H. Thaler, premio Nobel de Economía 2017, Estudios económicos, 35 (71),101-113.

* Centro Universitario de Ciencias Económico Administrativas. Universidad de Guadalajara, México. Correos electrónicos: jvargas2006@gmail.com,jgvh0811@yahoo.com,josevargas@cucea.udg.mx 


\begin{abstract}
This paper aims to analyze the main contributions of Richard $\mathrm{H}$. Thaler to the science of behavioral economics, which have been considered to grant him the Nobel Prize Sveriges Riksbank of Economics in 2017. His contributions incorporate psychology into the economic decision-making processes departing from instrumental rationality and the theory of rational choice to contribute to the generation of a new perspective in economic theory and economic policy. The method used is fundamentally analytical-descriptive based on a review of his main publications. Finally, it is concluded that Thaler and his collaborators exert a relevant influence when generating a new frame of reference to explain and predict economic results from an approximation of limited rational behavior, the theory of limited accounting and the model of planer-maker under the concept of nudge which have an impact on the design and implementation of public policies.
\end{abstract}

JEL Code: B31, B41, D91.

Keywords: Nudge, Behavioral Economics, Endowment effect, mental Accounting, Limited Rationality.

\title{
INTRODUCCIÓN
}

Richard Thaler ha sido galardonado con el Premio Sveriges Riksbank en memoria de Alfred Nobel por sus contribuciones a la economía del comportamiento. Estas contribuciones se resumen en la generación de conocimientos que incorporan la psicología en la teoría económica y la política económica mediante los análisis de cómo las personas toman decisiones económicas, a veces rechazando la racionalidad. Cuando le fue anunciado que ganaba el Nobel, mencionó que gastaría el monto tan irracionalmente como le fuera posible, en tanto que Cass Sunstein, coautor de uno de sus trabajos, señaló que esa acción era una elección racional que no tiene límites para el Nobel.

La economía neoclásica dominante se basó en el supuesto simplificado del comportamiento racional de las personas. Se asume, como modelo simplificado, que los individuos se comportan como agentes racionales cuando toman decisiones sobre la base de toda la información que tengan disponible. Sin embargo, el comportamiento económico de los seres humanos es un mecanismo que es complejo y se aleja mucho de la racionalidad instrumental que durante siglos se sustentó como premisa. Hoy se acepta que, si bien la toma de decisiones económicas de los individuos se guía por el interés propio, cada individuo es diferente considerando 
la racionalidad, la cual es limitada principalmente por las capacidades cognitivas, el autocontrol y la voluntad, además de las preocupaciones por la equidad y justicia.

La incorporación de conocimientos de la psicología a la economía neoclásica ha dado lugar a la economía conductual (Rabin, 1998; Camerer y Loewenstein, 2004; Dellavigna, 2009; Camerer, 2014).

\section{ECONOMÍA CONDUCTUAL: THALER, EL PREMIO NOBEL DE ECONOMÍA 2017}

El acercamiento de la economía conductual a la ley y la economía fue propuesto por Jolls, Sunstein y Thaler (1998). Thaler incorpora conocimientos de psicología para explicar por qué las personas se comportan de una forma que no son totalmente racionales. El trabajo de Thaler es fundamental para avanzar en los análisis del comportamiento económico de una forma más reconocible porque incorpora las limitaciones cognitivas, las percepciones de justicia y las preferencias sociales, así como el autocontrol individual en los procesos de toma de decisiones.

Una premisa básica de las teorías de Thaler es que para tener una buena economía hay que tomar en cuenta que la gente es humana. Con sus investigaciones, Thaler ha demostrado que el comportamiento humano todavía se puede anticipar, aunque se aparte de la racionalidad de manera consistente. Thaler ha elaborado un marco teórico, conceptual y empírico con la incorporación de nuevos conocimientos de psicología al análisis económico para desarrollar instrumentos analíticos y experimentales de predicción del comportamiento económico.

Desde la publicación de su trabajo "Hacia una teoría positiva de la elección del consumidor" en 1980 y en otras publicaciones posteriores ha analizado las variables de la psicología humana que influyen en el comportamiento económico para la toma de decisiones. Estas variables han sido llamadas "los tres límites", que son racionalidad limitada, fuerza de voluntad limitada y limitado interés propio (Thaler, 2015, p. 258).

\section{RACIONALIDAD LIMITADA}

La premisa que sustenta la racionalidad limitada es la dificultad para encontrar una solución óptima para maximizar la utilidad esperada de por vida en los procesos de toma de decisiones (Simon, 1955) que Thaler (1980) reemplaza por la 
simplificación de un conjunto de restricciones autoimpuestas. La teoría prospectiva es aplicada a la economía primeramente por Thaler (1980), quien muestra lo importante que son los puntos de referencia y la aversión a la pérdida en los entornos deterministas. En su trabajo de tesis doctoral, Thaler estima el valor de una vida estadísticamente experimentando con preguntas hipotéticas para determinar el valor de las reducciones del riesgo de mortalidad (Thaler, 1974; Thaler y Rosen, 1976).

\section{EL EFECTO DE DOTACIÓN}

En uno de sus primeros hallazgos de sus investigaciones, Thaler (1980) encontró que las personas asignan un mayor valor a las posesiones propias. El efecto de dotación es el término acuñado para describir la tendencia individual a valorar los bienes que uno posee en una forma más justa en relación con la aversión a perderlos (Kahneman y Tversky, 1979, Thaler, 1980). La aversión a las pérdidas explica por qué las personas valoran más el mismo bien cuando lo poseen que cuando no lo tienen. El efecto de dotación explicado en términos de aversión al riesgo (Thaler, 1980) se sustenta en el supuesto de que los individuos maximizan sus preferencias, que además dependen de la dotación como punto de referencia.

Un bien es mejor valorado cuando es parte de la dotación de un individuo que cuando no lo es. Thaler (1980) analiza escenarios donde el precio de compra de un bien o servicio es considerablemente más bajo que el precio al que la misma persona lo vendería. El autor explica este fenómeno apelando a la teoría de la perspectiva, considerando las razones por las que dar un bien es percibido como pérdida. En este sentido, observa que los individuos, adversos a la pérdida, valoran más los objetos que poseen que los mismos objetos que no son de su propiedad.

El efecto de dotación implica una diferencia entre los costos de bolsillo que los individuos consideran como pérdidas ponderadas fuertemente, mientras que los costos de oportunidad son considerados más ligeros. La aversión a las pérdidas hace que los individuos tiendan a permanecer más en el statu quo que Thaler analiza en los planes de pensión. En un debate sobre la robustez de estos hallazgos, Kahneman, Knetsch y Thaler (1990) probaron que el efecto de dotación es efectivamente robusto. 


\section{LA CONTABILIDAD MENTAL}

Posteriormente, Thaler $(1985,1999)$ desarrolló la teoría de la contabilidad mental. El fenómeno de la contabilidad mental se sustenta en el supuesto de que los individuos gastan su dinero en forma diferente si el gasto se etiqueta para un propósito específico. Los individuos racionalmente limitados adoptan sistemas de control interno para organizar y evaluar sus gastos y el ahorro. La teoría psicológica de la contabilidad mental (Thaler, 1985, 1999) trata de analizar cómo la cognición limitada afecta el gasto, el ahorro y otros comportamientos del hogar porque se cuestiona cómo toman decisiones las personas acerca del dinero. Esta teoría se relaciona con el control autolimitado como estrategia y con la equidad a través de la utilidad de transacción.

La teoría de la contabilidad mental simplifica la comprensión de las operaciones que utilizan los individuos a pesar de las limitaciones cognitivas para organizar y evaluar sus actividades económicas. La teoría de la contabilidad mental analiza la simplificación de las decisiones financieras por los individuos que se enfocan en el impacto estrecho más que en su efecto global. Las cuentas mentales se usan como una forma de racionalidad limitada de los individuos para simplificar sus procesos de decisiones financieras, en donde a cada cuenta se le asigna un presupuesto y su punto de referencia separado (Thaler y Sunstein, 2008, pp. 53-54). El valor que se atribuye a una cantidad de dinero depende de la cuenta asignada, del contexto y la situación. Mantener cuentas separadas para diferentes gastos protege de gastar más (Thaler, 1985).

De acuerdo a la hipótesis de la edición hedónica, es difícil para las personas integrar las pérdidas (Thaler, 1999). La teoría de los puntos de referencia se conecta con la teoría de las preferencias sociales. El componente asociado al consumo es separado del componente asociado a la transacción (Thaler, 1985), por lo que separa las fuentes de consumo en un componente relacionado con el consumo del producto o servicio, llamado la utilidad de adquisición, y otro componente vinculado con la transacción de compra-venta, llamado la transacción de utilidad. El precio de referencia se determina por lo que es equitativo para ambas partes en una transacción de acuerdo con su percepción de equidad (Thaler, 1985; Kahneman, Knetsch y Thaler, 1986a, 1986b).

Una de las implicaciones es que los que formulan las políticas públicas pueden empujar a los individuos a gastar el dinero etiquetado para propósitos y artículos específicos, incluso cuando no hay restricciones en la práctica de cómo gastar el dinero. Esta teoría de la cuenta mental tiene implicaciones para las transacciones 
en activos financieros (Shefrin y Statman, 1985; Thaler, 1999), sobre todo cuando se evalúan y experimentan las pérdidas y ganancias después de cerrar una cuenta mental por la pérdida o venta de un activo. Al cierre de una cuenta mental, los inversionistas tienden a vender en mayor medida los valores que han incrementado su precio que aquellos que han decrecido en su precio, porque su venta implica la experiencia de una pérdida.

En la investigación de Shafir y Thaler (2006) sobre el acoplamiento de individuos que recolectan vino, las compras por adelantado se consideran inversiones y el consumo de un bien adquirido antes y utilizado es codificado como libre y como ahorro. El desacoplamiento del gasto y el consumo reduce el dolor de compra como edición hedónica.

Los autores estudiaron el caso hipotético de la compra de un costoso boleto para un juego de básquetbol, en el que, para ir, los interesados están dispuestos a sufrir las inclemencias de una tormenta de nieve en tanto deben cerrar una cuenta mental sin pérdida. Sin embargo, si se les dice a los participantes que el boleto fue un regalo, entonces no hay percepción de pérdida en caso de que decidan permanecer en casa. En otro caso, estudiaron a los taxistas, quienes trabajan abriendo una cuenta mental separada para cada día y sufren de aversión de pérdida si no logran su objetivo diario (Thaler y Johnson, 1990).

El efecto dinero de la casa ocurre cuando los individuos obtienen una ganancia que es puesta en una cuenta mental y que los convierte en buscadores de riesgos a pesar de que tienden a ser adversos al riesgo. El efecto de punto de equilibrio es la tendencia a tratar de recuperar una pérdida asumiendo un comportamiento de búsqueda de riesgo (Thaler y Johnson, 1990, confirmado por Post et al., 2008).

\section{FUERZA DE VOLUNTAD LIMITADA}

La fuerza de voluntad limitada o autocontrol pobre limita la capacidad de los individuos para ahorrar para su jubilación. Por esta razón, los individuos no ahorran lo que se proponen para su vejez o para decisiones saludables de su estilo de vida. Existen tensiones entre la planificación a largo plazo y el hacer a corto plazo, por eso los propósitos de año nuevo son difíciles de sostener.

En el modelo de planeador-hacedor (Thaler y Shefrin, 1981; Shefrin y Thaler, 1988) el individuo tiene dos facetas. Como planeador maximiza la utilidad de por vida a valor presente descontado y como hacedor solo le interesa la utilidad 
actual. El planeador hace los juicios enfatizando el aspecto voluntario en donde los individuos toman la decisión. El modelo del planeador parte del supuesto de la neurociencia que considera que el cerebro humano es una colección de sistemas interactuantes que no siempre trabajan perfectamente juntos; por lo tanto, el comportamiento no es totalmente racional ni se basa siempre en preferencias y creencias consistentes (Kurzban, 2012; Shefrin y Thaler, 1988).

Fueron Thaler y Shefrin (1981) los primeros en presentar un modelo dual de autocontrol. La falta de autocontrol y la cognición limitada impiden que los agentes que elaboran sus planes sean los óptimos para quienes los ejecutan. Estos comportamientos se explican en un modelo dual o de dos sistemas que Thaler y Shefrin denominaron planificador-hacedor. Los autores tratan el problema de autocontrol como un problema de la teoría del principal agente, en donde el planeador trata de incentivar o constreñir al hacedor para maximizar la utilidad de vida.

En el comportamiento de compra y ahorro, los planificadores consideran la utilidad de por vida mientras que los hacedores son agentes miopes que solo evalúan las opciones para utilidad actual, como en el caso de incrementar los ahorros para el retiro (Thaler y Benartzi 2004). Thaler y Benartzi (2004) diseñan mecanismos que incrementan los planes de pensión eliminando los problemas de autocontrol. Entre las implicaciones, propone el esquema de "ahorrar más mañana" que consiste en dar oportunidades a los trabajadores para que se comprometan a contribuir en su propio plan de ahorro o de pensión. En el experimento del descuento hiperbólico, Thaler (1981) encontró patrones de descuento: es más pronunciado en el presente y futuro cercano que en un futuro lejano, las ganancias se reducen más que las pérdidas y los pequeños resultados se descuentan más que los grandes resultados.

\section{ECONOMÍA DEL “EMPUJÓN” O NUDGE}

Cass Sunstein en coautoría con Tahler publicaron Nudge. La economía del "empujón" o de la "ayudadita" (nudge) es expuesta en esta obra, en donde analizan la forma de otorgar incentivos a los individuos para tomar decisiones más racionales. El empujón (nudge) tiene efectos por defecto (default) en la toma de decisiones para el diseño de opciones, las cuales son especificadas previamente por la organización o agente que diseña la decisión, ya que muchos elijen una opción predeterminada. Thaler propuso cambiar la decisión por defecto (default) en definir las contribuciones a los planes de pensión (Thaler, 1994) juntando una tasa de ahorro y estrategia de inversión. Las personas que pueden asignar sus ahorros de pensión tienden a seguir una ingenua estrategia $1 / \mathrm{N}$ en la cual asignan sus ahorros 
entre los fondos disponibles, lo que trae efectos económicos no deseados. Benartzi y Thaler (2013) hicieron una estimación de las personas con planes de pensión con escala automática en los Estados Unidos y más recientemente en el Reino Unido (Thaler, 2015).

Esta perspectiva es considerada como paternalismo libertario (Thaler y Sunstein, 2003), porque empuja políticas que ayudan a los individuos a tomar mejores decisiones económicas. Thaler es un proponente del paternalismo libertario (Thaler y Sunstein, 2003, 2008; Sunstein y Thaler, 2003) que considera que los cambios benéficos en conducta se logran con el mínimo de políticas invasivas que empujan a las personas a tomar las decisiones correctas. El paternalismo libertario es un paternalismo relativamente débil y suave que se fundamenta en el supuesto de que el Estado puede ayudar a los ciudadanos a tomar las decisiones que hubieran elegido con una voluntad no viciada. Este argumento analizado desde las evidencias de la irracionalidad que ofrecen Thaler y Sunstein ha sido muy cuestionado por su debilidad. En efecto, el paternalismo libertario es cuestionado por no ser libertario, ya que elegir no significa que se tenga la libertad de tomar decisiones con autonomía (White, 2013).

El empujón (nudge), como un sistema de cambio, puede ayudar a los individuos a lograr un mejor autocontrol que les permita ahorrar para una pensión. Sin embargo, no todas las intervenciones libertarias paternalistas son deseables, por lo que Thaler y Sunstein (2008) limitan su recomendación solamente a las políticas que influyen en las decisiones de una manera que hace que los que eligen se sientan mejor, como juzgado por ellos mismos.

Los formuladores de políticas han sido influenciados por el empujón (nudge), tanto en Estados Unidos como en Inglaterra, en áreas como salud, planes de pensiones y otras que tienen consecuencias a largo plazo (Thaler, 2015). Idealmente, las políticas son probadas y evaluadas en lo que Thaler (2015, p. 338) denomina 'políticas basadas en evidencias'.

\section{LIMITADO INTERÉS PROPIO. PERCEPCIÓN DE LA JUSTICIA Y LA EQUIDAD}

Las personas se preocupan mucho por la justicia y la equidad penalizando los comportamientos que perciben como injustos. Las preferencias sociales y preocupaciones por la imparcialidad afectan las decisiones económicas en los mercados de consumo y laboral (Kahneman, Knetsch y Thaler, 1986a, 1986b). 
El trabajo de Thaler (1980) establece la justicia como un tema importante de la economía y provee evidencias empíricas de su importancia en las decisiones de consumo. La justicia percibida determina la utilidad de transacción en la teoría de la contabilidad mental (Thaler, 1985). Sus hallazgos confirman el supuesto de que la justicia limita la maximización de utilidades. Las preferencias respecto de la justicia en las interacciones entre los individuos se manifiestan de tres formas: hay individuos que se comportan de manera justa en configuraciones anónimas, hay individuos que usan recursos para penalizar conductas injustas contra ellos $\mathrm{y}$, por último, hay individuos que usan recursos para penalizar conductas injustas dirigidas a otros.

La investigación de Kahneman, Knetsch y Thaler (1986b) provee evidencias sobre la justicia percibida en los mercados de consumo. Detectaron tres determinantes de actitudes de justicia de las acciones de compañías: la transacción de referencia que negociar en precios y salarios, el código de acción y la ocasión para la acción. La investigación confirma que los precios se evalúan en un nivel de referencia, y que los cambios en los precios son relevantes. También encontraron que la ocasión que desencadena la decisión de fijación de precios influencia la equidad percibida. El consumidor acepta un incremento de los precios si hay un incremento en los precios de los insumos, pero no lo acepta si se debe al poder del mercado (Kahneman, Knetsch y Thaler, 1986b). Las percepciones de justicia se relacionan con el efecto dotación en donde los compradores y vendedores se sienten con derechos a los términos de intercambio acostumbrados y tratan cualquier deterioro como una pérdida (Thaler 2015, p. 131).

En tiempos de recesión económica, los empleados consideran injustas las reducciones salariales para mantenerse compatible con la demanda, por lo que prefieren eliminar algunos puestos de trabajo. Los recortes nominales al salario son percibidos como injustos (Kahneman, Knetsch y Thaler, 1986b).

En este caso el salario real decrece sin que la causa sea la inflación, por lo que se considera una actitud injusta de un abuso de la autoridad. Este comportamiento se explica con el experimento del juego del dictador (Kahneman, Knetsch y Thaler, 1986a), en donde aparentemente no todos los individuos maximizan su pago monetario, incluso en interacciones anónimas y sin consideraciones reputacionales. Sin embargo, el dictador, o sujeto que divide el dinero puede dividir la dotación más que ser forzado a elegir entre dos diferentes asignaciones como en Kahneman, Knetsch y Thaler (1986a). En una segunda parte del experimento incluyeron el juego del ultimátum donde el primer jugador propone una dotación que el segundo jugador acepta o rechaza. Si la propuesta se acepta los dos logran 
pagos y si se rechaza no obtienen pagos. Lo que encontraron los investigadores es que la propuesta era cercana a partes iguales (Kahneman, Knetsch y Thaler 1986a) $\mathrm{y}$, por lo tanto, se perciben como más justas.

\section{PSICOLOGÍA INVERSORA Y COMPORTAMIENTO FINANCIERO}

Thaler junto con Robert Shiller (2012) son considerados los fundadores del comportamiento financiero y la psicología inversora. En sus trabajos enfatizan que la irracionalidad no es suficiente para afectar los precios (Barberis y Thaler, 2003). Junto con Schiller, Thaler promueve un taller en el National Bureau of Economic Research (NBER). Las investigaciones de Thaler en esta área se han enfocado en investigar las implicaciones de la psicología del inversionista y en señalar las violaciones de la ley en los precios de los mercados financieros señalando los límites del arbitraje. Thaler analiza evidencias que demuestren las violaciones a la eficiencia de mercado en su trabajo sobre precios erróneos. Ha introducido modelos de psicología del inversionista para explicar la predictibilidad de los precios de las acciones y de la prima de capital, la desviación de los precios de los fundamentos y la reconciliación con la eficiencia del mercado y la racionalidad del inversionista.

La prima de capital en finanzas conductuales se explica con el estrecho bracketing (paréntesis) y la aversión a la pérdida que depende del punto de referencia y del cierre de la contabilidad de cuentas. La aversión al riesgo explica la prima de capital, si el período de evaluación es anual (Benartzi y Thaler, 1995). Esta miope aversión a la pérdida se confirma en otros trabajos (Thaler et al., 1997; Benartzi y Thaler, 1999). El estrecho paréntesis (bracketing) (Barberis, Huang y Thaler, 2006) explica por qué una fracción pequeña de hogares participa en el mercado de valores, lo que se conoce como el rompecabezas del mercado de valores.

El supuesto inherente al modelo financiero tradicional es cuestionado por Bondt y Thaler (1985), quienes sostienen que los negociadores racionales tienen las creencias correctas, a la vez que encontraron que el portafolio de las acciones perdedoras supera al portafolio de las acciones ganadoras. Esta conclusión ha sido muy disputada, a pesar de demostrar la robustez de sus resultados. Lee, Shleifer y Thaler (1991) explican el rompecabezas de fondo cerrado basado en negociadores ruidosos que tienen creencias incorrectas que sobreestiman o subestiman los retornos esperados relacionados con las expectativas racionales. Lamont y Thaler (2003) evidencian los casos en que se viola la ley de un precio examinando los datos de la equidad de esculpir (carve-outs), en los que una sociedad matriz vende la participación de una filial en el mercado de valores público y anuncia la intención 
de derivar el resto de las acciones de la de la filial. La razón para limitar el arbitraje es la dificultad de vender en corto las acciones esculpidas (carveout) a sobreprecio.

\section{CONCLUSIÓN}

La influencia que Thaler y sus colaboradores tienen en la integración de la psicología con la economía es relevante al aportar nuevas ideas y nuevos marcos de referencia para analizar los fenómenos económicos desde la perspectiva psicológica y predecir resultados económicos. Entre otras contribuciones, han aportado un nuevo acercamiento al comportamiento racional limitado, además de la teoría de la contabilidad mental y el modelo de planeador-hacedor, con marcos de referencia del interés propio limitado, la fuerza de la voluntad limitada y las preferencias sociales desde la perspectiva de la justicia y la equidad. En cuanto a las políticas para ayudar a los individuos a tomar mejores decisiones, las propuestas de Thaler se fundamentan en las ideas de la conducta económica. Como Thaler expresó en una entrevista, el más importante impacto de su trabajo es el reconocimiento de que los agentes son humanos y las decisiones de dinero no suelen ser tomadas de forma estrictamente racionales.

\section{REFERENCIAS}

Barberis, N., Huang, M., \& Thaler, R. H. (2006). Individual Preferences, Monetary Gambles, and Stock Market Participation: A Case for Narrow Framing. American Economic Review, 96(4), 1069-1090.

Barberis, N., Huang, M., \& Thaler, R.H. (2003). A Survey of Behavioral Finance. In G.M. Constantinides, M. Harris, \& R. Stulz (Eds.), Handbook of the Economics of Finance, (pp. 1051-1121). North Holland, Amsterdam: Elsevier Science B.V.

Benartzi, S. \& Thaler, R. H. (1995). Myopic Loss-Aversion and the Equity Premium Puzzle. Quarterly Journal of Economics 110(1), 75-92.

Benartzi, S., \& Thaler, R. H. (1999). Risk Aversion or Myopia? Choices in Repeated Gambles and Retirement Investments. Management Science, 45(3), 64-381.

Benartzi, S. \& Thaler, R. H. (2013). Behavioral Economics and the Retirement Savings Crises. Science 339, 1152-1153.

Camerer, C. F. (2014). Behavioral Economics. Current Biology, 24(18), R867-R871.

Camerer, C. F., Babcock L., Loewenstein G., \& Thaler, R. H. (1997). Labor Supply of New York City Cab Drivers: One Day at a Time. Quarterly Journal of Economics, 112(2), 407-442. 
Dellavigna, S. (2009). Psychology and Economics: Evidence from the Field. Journal of Economic Literature, 47(2), 315-372.

Jolls, C., Sunstein C., \& Thaler, R. H. (1998). A Behavioral Approach to Law and Economics. 50 Stanford Law Review, 1471-1550.

Kahneman, D., Knetsch, J. L., \& Thaler, R. H. (1990). Experimental Tests of the Endowment Effect and the Coase Theorem. Journal of Political Economy, 98(6), 1325-1348.

Kahneman, D., Knetsch J. L., \& Thaler, R. H. (1986a). Fairness and the Assumptions of Economics. Journal of Business, 59(4), 285-S300.

Kahneman, D., Knetsch J. L., \& Thaler, R. H. (1986b). Fairness as a Constraint on Profit Seeking. American Economic Review, 76(4), 728-741.

Kahneman, D., \& Tversky, A. (1979). Prospect Theory: An Analysis of Decision under Risk. Econometrica, 47(2), 263-291.

Kurzban, R. (2012). Why Everyone (Else) Is a Hypocrite: Evolution and the Modular Mind. Princeton: Princeton University Press.

Lamont, O. A. \& Thaler, R. H. (2003). Can the Market Add and Subtract? Mispricing in Tech Stock Carve-outs. Journal of Political Economy, 111(2), 227-268.

Lee, C. M. C., Shleifer, A., \& Thaler, R. H. (1991). Investor Sentiment and the Closed-End Fund Puzzle. Journal of Finance, 46(1), 75-109.

Post T., van dem Assem M. J., Baltussen G., \& Thaler, R. H. (2008). Deal or No Deal? Decision Making under Risk in a Large-Payoff Game Show. American Economic Review, 98(1), 38-71.

Rabin, M. (1998). Psychology and Economics. Journal of Economic Literature, 36(1), 11-46.

Shafir, E. \& Thaler, R. H. (2006). Invest Now, Drink Later, Spend Never: On the Mental Accounting of Delayed Consumption. Journal of Economic Psychology, 27(5), 94-712.

Shefrin, H. M. \& Statman, M. (1985). The disposition to sell winners too early and ride losers too long: Theory and evidence. Journal of Finance, 40(3), 777-790.

Shefrin, H. M. \& Thaler, R. H. (1988). The Behavioral Life-Cycle Hypothesis. Economic Inquiry, 26(4), 09-643.

Shiller, R. J. (2012). Finance and the Good Society. Princeton, NJ: Princeton University Press.

Sunstein, C. R., \& Thaler, R. H. (2003). Libertarian Paternalism Is Not an Oxymoron. University of Chicago Law Review, 70(4), 1159-1202.

Thaler, R. H. (1974). The Value of Saving a Life: A Market Estimate. PhD thesis, University of Rochester. 
Thaler, R. H. (1980). Toward a Positive Theory of Consumer Choice. Journal of Economic Behavior and Organization, 1(1), 39-60.

Thaler, R. H. (1981). Some Empirical Evidence on Dynamic Inconsistency. Economics Letters, 8(3), 201-207.

Thaler, R. H. (1985). Mental Accounting and Consumer Choice. Marketing Science, 4(3), 199-214.

Thaler, R. H. (1994). Psychology and Savings Policies. American Economic Review, 84(2), 186-192.

Thaler, R. H. (1999). Mental Accounting Matters. Journal of Behavioral Decision Making, 12(3), 183-206.

Thaler, R. H. (2015). Misbehaving: The Making of Behavioral Economics. New York: W. W. Norton \& Company

Thaler, R. H., \& Benartzi, S. (2004). Save More Tomorrow TM: Using Behavioral Economics to Increase Employee Saving. Journal of Political Economy, 112(1), S164-S187.

Thaler, R. H., \& Johnson, E. J. (1990). Gambling with the House Money and Trying to Break Even: the Effects of Prior Outcomes on Risky Choice. Management Science, 36(6), 643-660.

Thaler, R. H., \& Rosen, S. (1976). The Value of Saving a Life. In: N.H. Terleckyj (ed.) Household Production and Consumption. New York: National Bureau of Economic Research.

Thaler, R. H., \& Shefrin, H. M. (1981). An Economic Theory of Self-Control. Journal of Political Economy, 89(2), 392-406.

Thaler, R. H., \& Sunstein, C. R. (2003). Libertarian Paternalism. American Economic Review Papers and Proceedings, 93(2), 175-179.

Thaler, R. H., \& Sunstein, C. R. (2008). Nudge: Improving Decisions about Health, Wealth, and Happiness. New Haven: Yale University Press.

Thaler, R. H., Tversky, A., Kahneman, D., \& Schwartz, A. (1997). The Effect of Myopia and Loss Aversion on Risk Taking: An Experimental Test. Quarterly Journal of Economics 112(2), 647-661.

White, M. D. (2013). The manipulation of choice: Ethics and libertarian paternalism. The Independent Review, 18(2), Fall 2013.

(C) 2018 por los autores; licencia otorgada a la revista Estudios económicos. Este artículo es de acceso abierto y distribuido bajo los términos y condiciones de una licencia Atribución-No Comercial 3.0 Unported (CC BY-NC 3.0) de Creative Commons. Para ver una copia de esta licencia, visite http://creativecommons.org/ licenses/by-nc/3 